\title{
Compuestos fenólicos y capacidad antioxidante presentes en tres variedades de berenjena cultivadas en el valle de Mexicali, Baja California
}

\author{
Phenolic compounds and antioxidant capacity presents three varieties \\ of eggplant grown in the Mexicali valley Baja California \\ Daniel González-Mendoza ${ }^{1 *}$, Rosalba Troncoso-Rojas², Carlos Ceceña Durán ${ }^{1}$, \\ Onecimo Grimaldo-Juárez ${ }^{1}$, Roberto Zamora-Bustillo ${ }^{3}$,Esaú Ruiz-Sánchez ${ }^{3}$
}

\begin{abstract}
RESUMEN
En el presente trabajo se evaluó la calidad bioquímica de tres variedades de berenjena (Versátil, Barcelona y Esculentum) con potencial de ser cultivadas en condiciones de invernadero en el valle de Mexicali. A partir de frutos maduros se determinó el contenido de compuestos fenólicos totales y capacidad antioxidante. Los resultados mostraron que la variedad Esculentum fue la que presentó mayor contenido de actividad antioxidante $(23,43 \mu \mathrm{g} / \mathrm{mL}$ de extracto) no así de compuestos fenólicos totales en donde la variedad Versátil $(8,13 \mu \mathrm{g} / \mathrm{mg}$ de peso fresco) fue la que mostró los mayores niveles de estos compuestos. Lo que nos indica que la actividad antioxidante podría estar influenciada por la presencia de otros compuestos bioactivos. No obstante, la presencia de compuestos bioactivos en frutos de berenjena representa un parámetro de calidad, lo que podría ser útil para incrementar su cultivo y valor de producción en el valle de Mexicali. Futuros estudios deben ser encaminados para la determinación de otros compuestos bioactivos que podrían contribuir en la actividad antioxidante de las variedades de berenjena evaluadas y su impacto en la salud del consumidor.
\end{abstract}

Palabras clave: salud, estrés oxidativo, berenjena, valle de Mexicali, antioxidantes.

\begin{abstract}
In the present study our evaluated the quality biochemistry of three varieties of eggplant (Versatil, Barcelona and Esculentum) with the potential to be grown in a greenhouse in the Mexicali Valley. Since ripe fruit respectively, was determined the content of total phenolic compounds and antioxidant capacity. The results showed that the variety Esculentum showed higher antioxidant activity $(23.43 \mathrm{mg} / \mathrm{ml}$ of extract) but not of total phenolic compounds where the Versatile was the one that showed the greatest levels of these compounds $(8.13 \mathrm{mg} / \mathrm{mg}$ fresh weight). Which indicates that the antioxidant activity could be influenced by the presence of other bioactive compounds. However, the presence of bioactive compounds in fruits of eggplant could be a quality parameter, which could help to increase their cultivation and production in the Mexicali Valley. Future studies should be directed to the identification of other bioactive compounds that could contribute to the antioxidant activity of eggplant varieties evaluated and its impact on consumer health.
\end{abstract}

Key words: health, oxidative stress, eggplant, Mexicali Valley, antioxidants.

\section{Introducción}

Baja California (BC), México, se caracteriza por su clima semidesértico con precipitaciones pluviales menores a $500 \mathrm{~mm}$, y en algunos casos como en las zonas próximas al río Colorado (eg., valle de Mexicali) se tienen precipitaciones menores a $50 \mathrm{~mm}$. No obstante aun con las limitaciones ambientales BC es una de las zonas agrícolas más productivas del país en las últimas décadas y particularmente a partir de los años ochenta, con un elevado volumen de exportación local e

\footnotetext{
1 Instituto de Ciencias Agrícolas de la Universidad Autónoma de Baja California (ICA-UABC). Carretera a Delta s/n C.P. 21705, Ejido Nuevo León, Baja California, México.

2 Centro de Investigación en Alimentación y Desarrollo, A.C., Dirección de Tecnología de Alimentos de Origen Vegetal. Hermosillo, Sonora, México

3 División de Estudios de Posgrado e Investigación, Instituto Tecnológico de Conkal. Conkal, Yucatán, México.

* Autor para correspondencia: danielg@uabc.edu.mx
}

Fecha de Recepción: 9 Enero, 2015.

Fecha de Aceptación: 9 Abril, 2015. 
internacional de productos hortícolas, siendo las zonas de mayor producción San Quintín y el valle de Mexicali en menor escala (Vázquez-Angulo et al., 2013). En este sentido, la producción de hortalizas bajo agricultura protegida en el valle de Mexicali representa una alternativa viable, ya que se puede tener un mejor control de las variables ambientales, limitar la presencia de plagas y el uso de plaguicidas y hacer más eficiente el uso del agua (Sengar y Kothari, 2008). No obstante, la presencia de nuevos competidores a nivel nacional o internacional en la producción de hortalizas de mayor demanda (eg., tomate), crea la necesidad en el productor de incursionar en la producción de nuevas hortalizas como la berenjena (Solanum melongena). La berenjena es un producto con demanda creciente en los Estados Unidos y en otros países debido a la presencia de compuestos bioactivos en los frutos con importante efecto hipocolesterolémico, presencia de fenoles y capacidad antioxidante (Sadilova et al., 2006), lo que favorece la reducción de especies reactivas de oxígeno o radicales libres y niveles de colesterol que pueden iniciar una serie de procesos degenerativos como enfermedades cardiovasculares y desórdenes neurodegenerativos (Migliore y Coppedé, 2009). Es el caso de la berenjena que al igual que otras hortalizas su participación en la producción agrícola del valle de Mexicali se ha ido incrementando. Representando una función importante en el entorno socioeconómico debido a su efecto en la generación de divisas y de empleos (Macías-Macías, 2010).

No obstante, en el valle de Mexicali no existen estudios disponibles encaminados a evaluar la presencia de compuestos fenólicos y capacidad antioxidante en frutos de berenjena como un parámetro de calidad para incrementar su cultivo y valor de producción en el valle de Mexicali. De tal forma que el objetivo del presente trabajo consistió en evaluar el contenido de compuestos fenólicos y actividad antioxidante de tres variedades de berenjena cultivadas en condiciones de agricultura protegida en el valle de Mexicali.

\section{Materiales y Métodos}

Las muestras evaluadas consistieron en diez frutos fisiológicamente maduros de tres variedades de berenjenas (Versátil, Barcelona y Esculentum), colectadas de los campos experimentales del Instituto de Ciencias Agrícolas de la UABC. Se seleccionaron cinco frutos de cada variedad sin daño físico aparente con tamaño y color uniforme. Posteriormente se procedió a tomar una mezcla compuesta de $1 \mathrm{~g}$ de la cáscara procedente de cinco frutos de cada variedad y mezclarlo con $3 \mathrm{ml}$ de metanol al $80 \%$ mediante el uso de un micropistilo. Las muestras fueron centrifugadas por 5 min a 5000 rpm, el sobrenadante fue colectado y almacenado en oscuridad a $-20^{\circ} \mathrm{C}$, hasta su empleo.

\section{Cuantificación de compuestos fenólicos totales}

La determinación de compuestos fenólicos totales se realizó mediante el método espectrofotométrico de Folin-Ciocalteu usando el ácido gálico como material de referencia (SilvaEspinoza et al., 2013). Para esto se preparó una disolución $1 \mathrm{~N}$ del reactivo de Folin Ciocalteu, por medio de una dilución 1:2 del reactivo comercial $(2 \mathrm{~N})$ en agua destilada; el reactivo se protegió de la luz y se colocó en refrigeración hasta su uso. La reacción consistió en mezclar $100 \mu \mathrm{l}$ del extracto metanólico de las frutas con $1 \mathrm{ml}$ del reactivo de Folin-Ciocalteu. Después de 3 min se le adicionó $1 \mathrm{ml}$ de $\mathrm{Na}_{2} \mathrm{CO}_{3}(10 \%)$ y se dejó en reposo por 30 min en oscuridad. Posteriormente las absorbancias de las muestras fueron medidas a $760 \mathrm{~nm}$ usando un espectrofotómetro (Thermo Scientific Genesys 20). El contenido de compuestos fenólicos totales fue expresado como mg de acido gálico por gramo de peso fresco.

\section{Evaluación de la actividad antioxidante}

La actividad antioxidante fue determinada mediante la reducción del $\mathrm{Mo}(\mathrm{VI})$ a $\mathrm{Mo}(\mathrm{V})$ por el extracto metanólico y la subsecuente formación de un complejo de fosfato/Mo de color verde en un ambiente ácido (Prieto et al., 1999). El análisis consistió en utilizar $0,1 \mathrm{ml}$ del extracto metanoólico de las muestras y combinarla con $1 \mathrm{ml}$ de la solución reagente (0,6 M ácido sulfúrico, $28 \mathrm{~mm}$ fosfato de sodio y $4 \mathrm{~mm}$ molibdato de amonio). Los tubos conteniendo las muestras fueron incubados en un block térmico a $95{ }^{\circ} \mathrm{C}$ por $90 \mathrm{~min}$. Después la muestra se enfrió a temperatura ambiente y se leyó la absorbancia a $695 \mathrm{~nm}$ (Thermo Scientific Genesys 20) contra un blanco (sin muestra metanólica). La capacidad antioxidante se expresó como equivalentes de ácido ascórbico $(\mu \mathrm{g})$ por mg de extracto. 


\section{Análisis estadístico}

El experimento fue realizado completamente al azar con tres repeticiones. Las variables se examinaron mediante un análisis de varianza (ANOVA). La comparación de medias se efectuó con la Prueba de Tukey. El análisis de los datos se realizó mediante el paquete estadístico Statistica AX versión 6.1.

\section{Resultados y Discusión}

Los resultados mostraron que la variedad de berenjena Esculentum mostró la mayor capacidad antioxidante $(23,43 \mu \mathrm{g} / \mathrm{ml}$ de extracto) respecto de las otras dos variedades Barcelona ( $6,4 \mu \mathrm{g} / \mathrm{ml}$ de extracto) y Versátil $(8,9 \mu \mathrm{g} / \mathrm{ml}$ de extracto) respectivamente (Figura 1). Estos valores muestran que estas variedades tienen una capacidad antioxidante superior a otras hortalizas como chile habanero en donde se ha observado valores máximos de 8 $\mu \mathrm{g} / \mathrm{ml}$ de extracto (Núñez-Ramírez et al., 2011). Esto es de gran importancia ya que la presencia de compuestos bioactivos presente en esta hortaliza podrían actuar como antioxidantes, lo que podría favorecer su consumo contribuyendo a la disminución de riesgos de desarrollar enfermedades crónicas no transmisibles y proporcionarle un valor agregado. Los compuestos fenólicos pueden interferir con el desarrollo de enfermedades degenerativas, ya sea mediante la activación de sistemas enzimáticos que participan en los procesos de desintoxicación de xenobióticos o inhibiendo la expresión de genes activadores de procesos carcinogénicos (Bafeel and Ibrahim, 2008). De tal forma que la presencia de estos compuestos en las tres variedades de berenjena evaluadas puede representar un valor agregado al producto. En este sentido la evaluación del contenido de compuestos fenólicos en las tres variedades de berenjena (Figura 2) mostraron que la variedad Versátil fue la que presentó mayor contenido de compuestos fenólicos, aproximadamente un $70 \%$ de contenido de fenoles totales respecto de las otras variedades. La concentración observada de compuestos fenólicos totales en la variedad versátil fue similar a lo observado en ciertos genotipos de tomatillo morado al ser evaluados en condiciones similares (González-Mendoza et al., 2010). No obstante, la presencia de una mayor cantidad de compuestos fenólicos no mostró una interacción positiva con una mayor actividad antioxidante (Figura 1). Lo que nos indica que la actividad antioxidante podría estar influenciada por la presencia de otros compuestos bioactivos. Similares resultados han sido observado anteriormente en frutos de tomatillo morado en donde se encontró que la cantidad de antocianinas no mostraban una correlación positiva con la actividad antioxidante de los frutos (González-Mendoza et al., 2010).

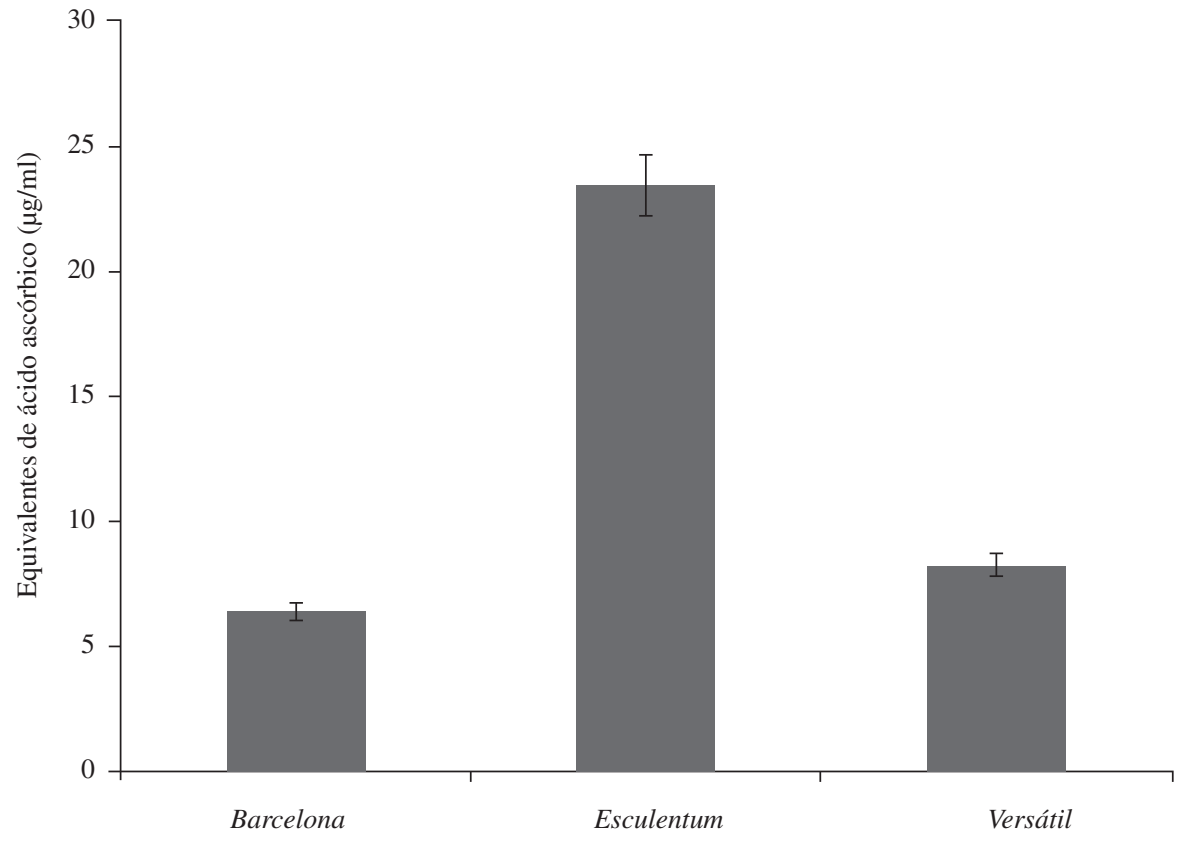

Figura 1. Actividad antioxidante de los extractos metanólicos de las tres variedades de berenjenas evaluadas. 


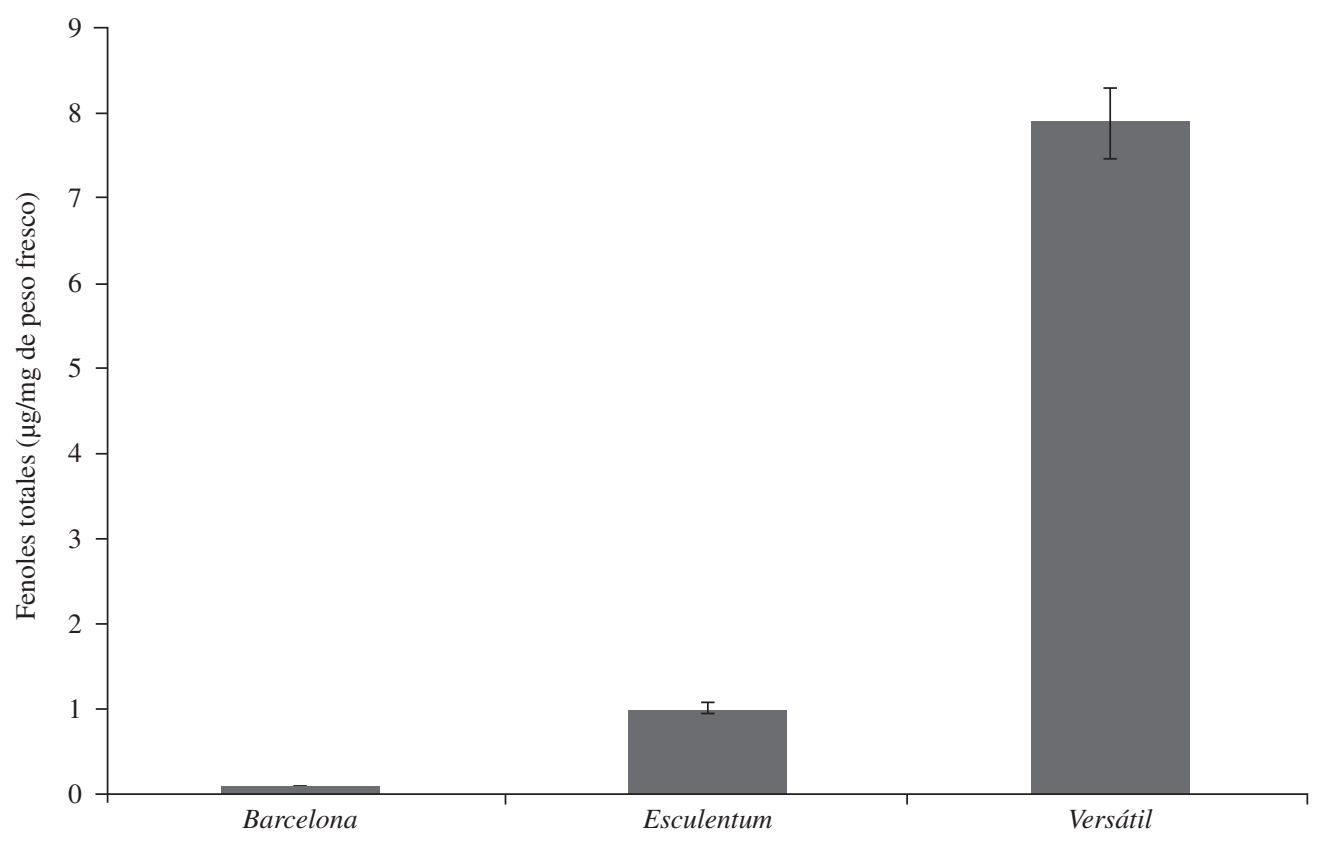

Figura 2. Compuestos fenólicos totales presentes en los extractos metanólicos de las tres variedades de berenjenas evaluadas.

\section{Conclusiones}

En el presente estudio se observó que las variedades de berenjena Versátil y Esculentum presentan compuestos bioactivos que pueden representar un potencial para incrementar su cultivo y valor de producción en el valle de Mexicali. Futuros estudios deben ser encaminados para la determinación de otros compuestos bioactivos que podrían contribuir en la actividad antioxidante de las variedades de berenjena evaluadas y su impacto en la salud del consumidor.

\section{Agradecimientos}

Se agradece el apoyo al Dr. Fidel Núñez por proporcionar el material vegetal y el apoyo recibido por la 18a Convocatoria Interna de Apoyo a Proyectos de Investigación de la Universidad Autónoma de Baja California.

\section{Literatura Citada}

Bafeel, S.O.; Ibrahim, M.M.

2008. Antioxidants and accumulation of $\alpha$-tocopherol induce chilling tolerance in Medicago sativa. International Journal Agriculture Biology, 10: 593-598.

González-Mendoza, D.; Grimaldo-Juárez, O.; Soto-Ortiz R.;

Escoboza-García, F.; Santiaguillo Hernández, J.F.

2010. Evaluation of total phenolics, anthocyanins and antioxidant capacity in purple tomatillo (Physalis ixocarpa) genotypes. African Journal Biotechnology, 9: 5173-5176.

Macías-Macías, A.

2010. Competitividad de México en el mercado de frutas y hortalizas de estados unidos de américa, 1989-2009. Agroalimentaria. 31:31-48.

Migliore, L.; Coppedè, F .

2009.Oxidative Stress and mechanisms of environmental toxicity. Mutation Research, 31: 73-74.
Núñez-Ramírez, F.; González-Mendoza, D.; Grimaldo-Juárez, O.; Díaz, L.C.

2011. Nitrogen fertilization effect on antioxidants compounds in fruits of habanero chili pepper (Capsicum chinense). International Journal Agriculture Biology, 13:827-830.

Prieto, P.; Pineda, M.; Aguilar, M.

1999. Spectrophotometric quantitation of antioxidant capacity through the formation of a phosphomolybdenum complex: specific application to the determination of vitamin E. Analytical Biochemistry, 269: 337-341.

Sadilova, E.; Stintzing, F.C.; Carle, R.

2006. Anthocyanins, colour and antioxidant properties of eggplant (Solanum melongena L.) and violet pepper (Capsicum аппиит L.) peel extracts. Zeitschrift für Naturforschung. C. 61:527-35. Sengar, S.H.; Kothari, S.

2008. Economic evaluation of greenhouse for cultivation of rose nursery. African Journal of Agricultural Research, 3: 435-439. 
Silva-Espinoza, B.A.; Ortega-Ramírez, Luis A.; González-Aguilar, Gustavo A.; Olivas, Isela; Ayala-Zavala, Jesús F.

2013. Protección antifúngica y enriquecimiento antioxidante de fresa con aceite esencial de hoja de canela. Revista Fitotecnia Mexicana, 36:217-224.
Vázquez-Angulo, J.C.; Grimaldo-Juárez, O.; GonzálezMendoza, D.

2013. Producción de Cucumis sativus en el valle de Mexicali, Baja California, México. Idesia (Arica), 3:17-20. 
